\title{
Applied Magnetic Resonance Under Conditions of a Pandemic
}

\author{
Vladimir I. Chizhik ${ }^{1} \cdot$ Murat S. Tagirov $^{2}$
}

Published online: 22 October 2020

(c) Springer-Verlag GmbH Austria, part of Springer Nature 2020

The preparation of this special issue coincided with the COVID 2019 pandemic period. This circumstance has given a reflex in two aspects: first, the scientists did not interrupt their research and its presentation to the scientific community (moreover, many articles are submitted by international or inter-university scientific teams), and second, the methods presented in the issue may be used, directly or indirectly, in the fight against the pandemic (in the issue, more than half of the articles deal with medical/biological topics or related problems).

One of the most important achievements of NMR applications is magnetic resonance imaging (MRI) - an interdisciplinary field of science at the intersection of physics, chemistry, computer science, engineering, and medicine. Despite the numerous advantages and achievements of the modern MRI methods, there exists justified searching new methodological techniques. One of the promising fields in MRI diagnostics is the mapping of distribution and state of nuclei other than ${ }^{1} \mathrm{H}$. In the article, "New aspects of biodistribution of perfluorocarbon emulsions in rats: thymus imaging", Yu. A. Pirogov and co-workers show that MRI on fluorine-19 nuclei is an effective tool for imaging and monitoring phagocytic cells (macrophages, stem cells, etc.). The ${ }^{19} \mathrm{~F}$ MRI technique is not just a way of visualizing certain organs, but it is also an assessment of the state of the immune system. The thymus is one of the central organs of the immune system, and information obtained using ${ }^{19} \mathrm{~F}$ MRI about the behavior of this organ in response to infection provides a better understanding of the role of the thymus in the immunology of infectious diseases.

In various application areas, it becomes necessary to analyze several expert orderings for the optimization of clustered rankings of examination objects. In particular, it is important in the NMR-based metabolomics, which is a rapidly developing

Vladimir I. Chizhik

v.chizhik@spbu.ru

Murat S. Tagirov

Murat.Tagirov@kpfu.ru

1 Saint Petersburg State University, Saint Petersburg, Russia

2 Kazan Federal University, Kazan, Russia 
discipline for early diagnosis and biomarker discovery in clinical applications. In the article, "Kemeny-Snell distance in nuclear magnetic resonance metabolomics", MinJi Shina, Tarmo Veskiojaa, Tiina Titmaa, and Ago Samoson suggested to use the new Kemeny-Snell distance (KSD) metric for metabolomics and validated results with partial least squares-discriminant analysis. The KSD metric allows the identification of the most relevant chemical shift ranges directly from ${ }^{1} \mathrm{H}$ spectra without metabolite library. The specific goal of the study is to limit the set of variables used in the KSD calculations so that non-important variables would not introduce excessive noise into the KSD data. The authors' results may be considered as a new step towards the applicability of the NMR metabolomics in medical diagnosis.

Many diseases are associated with the existence of molecular aggregates. The polymerization (aggregation) of the hemoglobin $\mathrm{S}(\mathrm{HbS})$ is the basic molecular process of worldwide distributed sickle cell disease. The nuclear magnetic relaxation plays an important role in the investigations of molecular mobility in various objects and, basing on this method, it has earlier been concluded that the sickle cell disease is accompanied by the increase in the rotational correlation time of water molecules binding to the $\mathrm{HbS}$ during the aggregation process. The rotational correlation time was earlier experimentally estimated from the investigations of ${ }^{1} \mathrm{H}$ relaxation at different resonance frequencies considering the single magnetic relaxation mechanism, namely the dipole-dipole interaction. In the article, "Assessment of contribution of Curie-Spin mechanism in proton relaxation during aggregation process of hemoglobin S", C. Cabal, M. Lores, V. I. Chizhik, S. O. Rabdano, and J. C. García-Naranjo indicate that it is necessary to consider the possible influence of the Curie-Spin relaxation mechanism on the proton relaxation in the presence of $\mathrm{HbS}$ aggregates, that may be manifested in the case of slow molecular motion and in experiments with high magnetic fields.

Characterization of state and stability of protein solutions is of great importance for biochemistry/biophysics and medicine. Particularly, the monitoring of the protein aggregation is crucial at all stages of biotechnological production and in diagnosis of dangerous diseases. Usually proteins are dissolved in buffers containing water and salts. The goal of the work of S. O. Rabdano, S. S. Bystrov, D. A. Luzik, and V. I. Chizhik "NMR relaxation of nuclei of buffer as a probe for monitoring protein solutions including aggregation processes" is to study of prospects and possibilities of NMR relaxation of solvent (buffer) nuclei for monitoring the state of proteins in solutions. The relaxation rates of the solvent nuclei $\left({ }^{1} \mathrm{H},{ }^{2} \mathrm{H},{ }^{23} \mathrm{Na},{ }^{35} \mathrm{Cl}\right)$ in solution of soluble and aggregated RRM 2 domain of TDP-43 protein were studied and the special case of aggregation induced by mild oxidative stress, using treatment by hydrogen peroxide, was considered. The relaxation rates of solvent nuclei for systems, containing components like the RRM2 domain, are sensitive to the presence of proteins even at low protein concentrations, and the relaxation rates of different nuclei reflect various aspects of the state of proteins in solutions.

Investigation of model homopeptides is an important step for understanding of structure and NMR properties of more complex organic substances like branched peptides based on lysine monomers, e.g., dendrimers, dendrigrafts and dendritic polymer brushes. Such a question is studied in the work "Molecular dynamics and spin-lattice NMR relaxation in $\alpha$ - and $\varepsilon$-polylysine" by V. V. Bezrodniy, O. 
V. Shavykin, S. E. Miktaniuk, I. M. Neelov, and D. A. Markelov. The $\alpha$-polylysine is widely used as a cell culture substratum to increase the adhesion of cells and $\varepsilon$-polylysine is commonly used as antimicrobial peptide and, in particular, as a food preservative. The authors have performed the molecular dynamics simulation (MD method) of two linear lysine peptides with the same number of lysine monomers but with different connections between them through $\alpha$ - and $\varepsilon$-peptide bonds. Using $\mathrm{MD}$, the orientational mobility of $\mathrm{CH}_{2}$ groups in both peptides can be characterized by second-order autocorrelation functions, which are used for the theoretical description of spin-lattice NMR relaxation rates. The results allow one to use NMR for discrimination between $\alpha$-lysine and $\varepsilon$-lysine peptides as well as between linear lysine peptides (or their mixtures) and lysine dendrimers. Besides, the results obtained in this article could also be used for better understanding of differences in structure and local mobility in lysine dendrimers, dendrigrafts, brushes and other branched lysine structure.

The article "Hydrodynamic fluctuations in liquids observed by NMR modulated gradient spin-echo method" by Janez Stepišnik is devoted to the study of the behavior of molecular velocity autocorrelation functions (VAFs). The VAF is a key quantity for the characterization of the molecular translation dynamics, which contains the information about underlying processes of molecular motion in fluids. The modulated gradient spin echo (MGSE) method provides a direct insight into the low-frequency part of the molecular velocity auto-correlation spectra in fluids. Because the MGSE method gives a spectrum that is time-averaged over the trajectory elapsed, the spin behavior is able to reflect local inhomogeneities in the initial interval after spin excitation. In the case of fluids, it manifests as an initial nonexponential decay of the spin echo signals, that can be attributed to the spatial heterogeneity of molecular self-diffusion due to the molecular motion in micro vortices of hydrodynamic oscillations. The author shows that the hydrodynamic fluctuations occur in water, ethanol, toluene, and also in a mixture of water with glycerol of low content, but they disappeared with increasing the glycerol concentration.

The results of the study of diffusion processes in solids using the NMR method are presented in the article "Anomalously high fluorine mobility in tysonite-like $\mathrm{LaF}_{3}: \mathrm{ScF}_{3}$ nanocrystals-NMR diffusion data" by L. B. Gulina, A. F. Privalov, M. Weigler, I. V. Murin, V. Tolstoy, and M. Vogel. Solid electrolytes based on inorganic fluorides become widely used in chemical sensors, voltaic cells and many other solid-state electrochemical devices. $\mathrm{LaF}_{3}$, crystallizing in tysonite structure, is a prospective ion-conducting solid electrolyte due to its inert nature and high F-conductivity. To investigate long range transport of fluorine ions, the authors applied the ${ }^{19} \mathrm{~F}$ static field gradient (SFG) NMR diffusometry, which had proved useful in their previous studies on bulk and nanostructured $\mathrm{LaF}_{3}$ materials. NMR diffusion studies show that homovalent substitution of $\mathrm{La}^{3+}$ by $\mathrm{Sc}^{3+}$ with a smaller ionic radius leads to around four orders of magnitude faster fluorine diffusion as compared with pure crystalline $\mathrm{LaF}_{3}$. The homovalent doping is a new route to improve the conductivity of tysonite structured nano-materials.

Rare-earth orthoferrites in whole and, in particular, $\mathrm{EuFeO}_{3}$ are of a relentless interest due to their promising properties for practical usage. Being potential materials for the finding of multiferroic behavior, these ferrites undergo attention concerning their synthesis, modifications and study of their properties. The article "Peculiarities of ${ }^{57} \mathrm{Fe}$ 
NMR Spectrum in Micro- and Nanocrystalline Europium Orthoferrites" by A. Sklyarova, V. I. Popkov, I. V. Pleshakov, V. V. Matveev, H. Štěpánková, and V. Chlan is devoted to these questions. Although $\mathrm{EuFeO}_{3}$ is known already quite a long time and this material in the bulk state has been well studied, since the beginning of nanomaterials era, the interest to this substance has renewed. NMR spectra of ${ }^{57} \mathrm{Fe}$ dispersed europium orthoferrite in powder samples with micro- and nanocrystalline particles have been studied for the first time. The presented NMR study of micro- and nano-powders of $\mathrm{EuFeO}_{3}$ shows the appearing of new properties in europium orthoferrites produced by glycine-nitrate combustion method. The obtained results point to the possible spin reorientation transition in the absence of an external field and the appearance of ferroelectric properties in the samples.

$\mathrm{Yu}$. Bunkov in the article "Magnonic superfluidity versus Bose condensation" tries to comprehend and interpret numerous works in this intriguing direction over the past few decades, discussing mainly two different coherent quantum phenomena of magnonic bosons: Bose-Einstein condensation (mBEC) and Superfluid State of Magnons (SSM). Magnon BEC is a quantum phenomenon determined by local density of bosonic quasiparticles. The superfluid state of magnons is a long-range coherent quantum state characterized by the rigidity of the order parameter. The author describes in detail the mechanism of the formation of SSM. In this state, the deflected magnetization can coherently precess even in a strongly inhomogeneous magnetic field. Much remains to be investigated, including by the magnetic resonance method, but this article will undoubtedly allow the deeper understanding of physics of the phenomenon and give promising practical applications of fundamental research.

In 2019, the world community honorably celebrated the 75th anniversary of the discovery of the EPR by Evgenij Zavoisky at the Kazan University. The article of P. Höfer and U. Eichhoff "75 years of EPR. EPR Milestones in 60 years Bruker history" is devoted to the history of development of EPR spectrometers, which was largely determined by the activities of Bruker Physik AG. Initially, EPR spectrometers were homebuilt and mostly dedicated to a special application but commercial instruments, on the contrary, should be universal and give the opportunity to implement a broad range of methods and applications. Therefore, they should be compatible with any valuable accessory, offer optimal sensitivity and resolution, they should be easily convertible to other frequency bands and adapted to new methods in investigations. All these most difficult tasks were solved with honor that allowed Bruker to become a world leader in the production of modern commercial EPR spectrometers.

We hope that the publications presented in this issue will attract the attention of scientific community and promote further cooperation between different scientific groups. The Coronavirus has been sweeping the globe but we believe that, as with any epidemic, the outbreak of Coronavirus will ever come to an end. We wish everyone health and successes in scientific activity. We would like to bring our gratitude to all authors who have contributed to this special issue.

Publisher's Note Springer Nature remains neutral with regard to jurisdictional claims in published maps and institutional affiliations. 